\title{
Simple Optical Methods for Measuring Optical Nonlinearities and Rotational Viscosity in Nematic Liquid Crystals
}

\author{
Gun Yeup Kim, and Chong Hoon Kwak \\ Yeungnam University \\ South Korea
}

\section{Introduction}

Knowing the nonlinear optical properties such as nonlinear refraction, nonlinear absorption and rotational viscosity in dye-doped nematic liquid crystals (NLCs) is crucially important for photonic applications like liquid crystal display (LCD). Versatile optical methods have been developed for measuring the nonlinear refraction and nonlinear absorption including third harmonic generation (deSouza et al., 1999), degenerate four wave mixing (Liao et al., 1998), optical Kerr gate (Gomes et al., 1996), nonlinear interferometry (Yavrian et al., 1999), ellipse rotation (Lefkiry et al., 1998), phase distortion method (Watanabe et al., 1995), among others. Although these techniques are sensitive, they require complicated experimental setup to measure the optical nonlinearities. The Z-scan technique (Sheik-Bahae et al., 1990), is a simple and highly sensitive single beam method that uses the principle of spatial beam distortion to measure both the sign and the magnitude of the optical nonlinearity. Nowadays, the $\mathrm{Z}$ scan method has been most popularly used.

To determine the rotational viscosity coefficient, various experimental methods have been extensively investigated which include: the light scattering measurement (Durand et al., 1995), capillary flow measurement (Gähwiller, 1971), rotating magnetic field technique (Prost \& Gasparoux, 1971), shear-waves reflectance technique (Kneppe et al., 1982; Martinoty \& Candau, 1971), nuclear magnetic resonance technique (Martins et al., 1986), capacitance method (Leenhouts, 1985) and transient current method (Imai et al., 1995). Among them most widely used techniques are capacitance method and transient current method. Capacitance method measures the time constant of an exponentially decaying director relaxation from a non-equilibrium to an equilibrium state in a twisted nematic cell upon switching on or off the applied voltage. Transient current method measures the peak amplitude of the transient current induced by a direct-current voltage pulse application.

In this chapter, we propose simple and accurate optical methods for determining the nonlinear refractive coefficient, the nonlinear absorption and the rotational viscosity coefficient in dye-doped nematic liquid-crystal and also present the theories developed. This chapter is organized as follows. In section 2, the theoretical backgrounds are provided for closed- and open- aperture Z-scan transmittance, knife-edge X-scan and modified Z-scan by switching on or off the applied electric field, taking into account two photon absorption. In

Source: New Developments in Liquid Crystals, Book edited by: Georgiy V. Tkachenko, ISBN 978-953-307-015-5, pp. 234, November 2009, I-Tech, Vienna, Austria 
section 3, a series of experiments are performed and the experimental results are discussed, and finally, conclusions are drawn in section 4.

\section{Theory}

\subsection{Closed and open aperture Z-scan theory for complex optical nonlinearity}

In this section, we present the optical method to determine the nonlinear refraction and nonlinear absorption by Z-scan technique. The nonlinear medium is scanned along the $\mathrm{z}$ axis in the back focal region of an external lens, and the far-field on-axis (i.e., closed aperture) transmittance and the whole (i.e., open aperture) transmittance are monitored as a function of the scan distance $z$. The open aperture Z-scan transmittance is insensitive to the nonlinear refraction and solely determines the nonlinear absorption, whilst the closed aperture Z-scan transmittance is coupled with both of the nonlinear effects. Actually, both the nonlinear refraction and the nonlinear absorption are often present simultaneously in nonlinear optical materials. Nonlinear absorption is inevitably present for resonant absorption wavelength ranges as well as for transparent regions owing to multi-photon absorption when the laser beam intensity is sufficiently high or because of other nonlinear processes. For simplicity, we only concentrate on two-photon absorption (TPA). Consider the fundamental Gaussian electric field (TEM ${ }_{00}$ mode) of travelling in the $z$ direction as $E(r, z)=E_{o} \frac{w_{o}}{w(z)} \exp \left[-\left(\frac{r^{2}}{w^{2}}+i \frac{k r^{2}}{2 R}\right)\right] \exp [-i \varphi(z)]=E(0, z) \exp \left[-i \frac{k r^{2}}{2 \tilde{q}}\right]$ where $\mathrm{E}_{0}$ is the amplitude of the electric field at the focus (i.e., $z=0), w(z)=w_{o}\left(1+\tilde{z}^{2}\right)^{1 / 2}$ is the beam radius at, is the beam at $z$, $w_{0}$ waist radius at focus, $R(z)=z\left(1+1 / \tilde{z}^{2}\right)$ is the radius of curvature of the wave front at $z, z, \tilde{z}=z / z_{o}$ is the dimensionless propagation distance, $z_{o}=k w_{o}^{2} / 2$ is the Rayleigh diffraction length of the beam, $k=2 \pi / \lambda$ is the wave number and $\lambda$ is the wavelength of laser beam. Here, $\tilde{q}$ denotes the complex beam parameter that contains all the information needed to specify its characteristics in the beam propagation and is defined by $1 / \tilde{q}=1 / R-i \lambda / \pi w^{2}$. For a Kerr medium with TPA process the nonlinear refractive index and the nonlinear absorption coefficient can be written as (Sheik-Bahae et al., 1990; Kwak et al., 1999)

$$
\begin{aligned}
& n=n_{o}+n_{2} I \\
& \alpha=\alpha_{o}+\beta I
\end{aligned}
$$

where $n_{0}$ is the linear refractive index, $n_{2}$ is the nonlinear refractive coefficient, $I(r, z)=\varepsilon_{0} c n_{o}|E(r, z)|^{2} / 2$ denotes the incident Gaussian laser beam intensity, and $\alpha_{0}$ is the linear absorption coefficient and $\beta$ is the two photon absorption coefficient. Solving the wave equation that describes the propagation of a Gaussian laser beam through the medium and neglecting the transverse effect, the intensity variation for two photon absorption and the nonlinear phase shift of the beam at the exit surface of the sample are given by, respectively, (Sheik-Bahae et al., 1990)

$$
I_{e}(r, z)=\frac{I(r, z) \exp \left[-\alpha_{o} L\right]}{1+q(r, z)}
$$




$$
\Delta \phi(r, z)=\frac{k n_{2}}{\beta} \ln [1+q(r, z)]
$$

where $I(r, z)=I_{0} \exp \left[-2 r^{2} / w^{2}\right] w_{0}^{2} / w^{2}$ is the incident beam intensity, $I_{0}$ is the on-axis intensity at focus, $q(r, z)=\beta I(r, z) L_{c f t}$ is the nonlinear absorbance of the medium, $L_{\text {eff }}=\left(1-\exp \left[-\alpha_{0} L\right]\right) / \alpha_{o}$ is the effective length of the sample, and $L$ is the sample thickness. By combining Eq.(2a) and Eq.(2b) we obtain the complex electric field at the exit surface of the sample:

$$
\begin{aligned}
E_{e}(r, z) & =\left[\frac{I(r, z) \exp \left[-\alpha_{o} L\right]}{1+q(r, z)}\right]^{1 / 2} \exp (i \Delta \phi) \\
& =E(r, z) \exp \left[i \Delta \phi\left(1+i \frac{\beta}{2 k n_{2}}\right)\right] \exp \left[-\alpha_{o} L / 2\right] .
\end{aligned}
$$

According to the aberration-free approximation of a Gaussian beam, which requires the Gaussian beam profile be approximated as being parabolic, by expanding the exponential in the intensity and retaining only the quadratic term, the nonlinear phase shift, Eq. (2b) can be approximated as:

$$
\Delta \phi(r, z) \cong-\frac{k n_{2}}{\beta} \frac{q_{o}}{1+q_{o}} \frac{2 r^{2}}{w^{2}}
$$

where $q_{o}=q(0, z)=q_{\infty} /\left(1+\tilde{z}^{2}\right)$ and $q_{o o}=\beta I_{o} L_{c f f}$. It is noted that Eq.(4) is always valid whether $|q| \geq 1$ or not. By substituting Eq.(4) into Eq.(3) and employing the complex beam parameter formulation (Kwak et al., 1999) we have finally obtain the closed aperture Z-scan transmittance of the far-field at the aperture plane, including both of the effects of nonlinear absorption and nonlinear refraction as follows:

$$
T_{\text {close }}(z)=\frac{1}{1-\frac{4(\tilde{z}-\eta)}{\left(1+\tilde{z}^{2}\right)^{2}\left(1+q_{0}\right)} \Delta \Phi_{0}+\frac{4\left(1+\eta^{2}\right)}{\left(1+\tilde{z}^{2}\right)^{3}\left(1+q_{0}\right)^{2}} \Delta \Phi_{0}^{2}}
$$

where $\Delta \Phi_{o}=k n_{2} I_{o} L_{\text {eff }}$ is the on-axis nonlinear phase at focus and $\eta=q_{o o} / 2 \Delta \Phi_{o}=\beta / 2 k n_{2}$ is the ratio of the imaginary part to the real part of the complex nonlinearity and is inversely proportional to the figure of merit (FOM), defined as $\mathrm{FOM}=n_{2} / \beta \lambda$ (Lenz et al., 2000). The nonlinear absorptive and refractive contributions to the closed aperture Z-scan transmittance are coupled in terms of $\eta$ or FOM. When the aperture is removed, however, the Z-scan is irrelevant to beam distortion caused by nonlinear refraction and is only a function of the nonlinear absorption, as mentioned above. Hence, the nonlinear absorption coefficient can readily be determined from the open aperture Z-scan transmittance. By spatially integrating Eq.(2a) at $z$ over all $r(0 \leq r \leq \infty)$ without having to include the free space propagation process, we have the $\mathrm{CW}$ open aperture $\mathrm{Z}$-scan transmittance as:

$$
T_{\text {open }}(z)=\frac{\ln \left[1+q_{o}(z)\right]}{q_{o}(z)}
$$


where $q_{o}=q_{\infty} /\left(1+\tilde{z}^{2}\right)$ and $q_{\infty}=\beta I_{o} L_{e f f}$. Once the nonlinear absorption coefficient $\beta$ or $q_{\infty}$ is unambiguously extracted from an open aperture Z-scan, one can use the closed aperture Zscan transmittance to determine the remaining unknown coefficient $n_{2}$ or $\Delta \Phi_{o}$ from Eq.(5).

\subsection{Knife-edge X-scan theory for nonlinear absorption}

In this section, we propose an alternative optical method for determining the nonlinear absorption coefficient, so-called knife-edge X-scan method. The knife-edge scanning technique is a simple single beam method for measuring a laser beam profile such as the beam radius and the radius of curvature of the wave front (Suzaki \& Tachibana, 1975). Due to its high accuracy, simple apparatus and easy to data analysis, the knife-edge scanning method has been widely used. As the knife-edge along the x-axis moves across the beam propagation direction, the beam power at the far-field gradually decreases and eventually goes to zero. For a Gaussian beam distribution, the (measured) beam power is given by integrating the Gaussian function from negative infinity to present knife-edge position and becomes the error function.

Figure 1 represents schematic diagram for the knife-edge $X$-scan method proposed in this work to determine the nonlinear absorption coefficient.

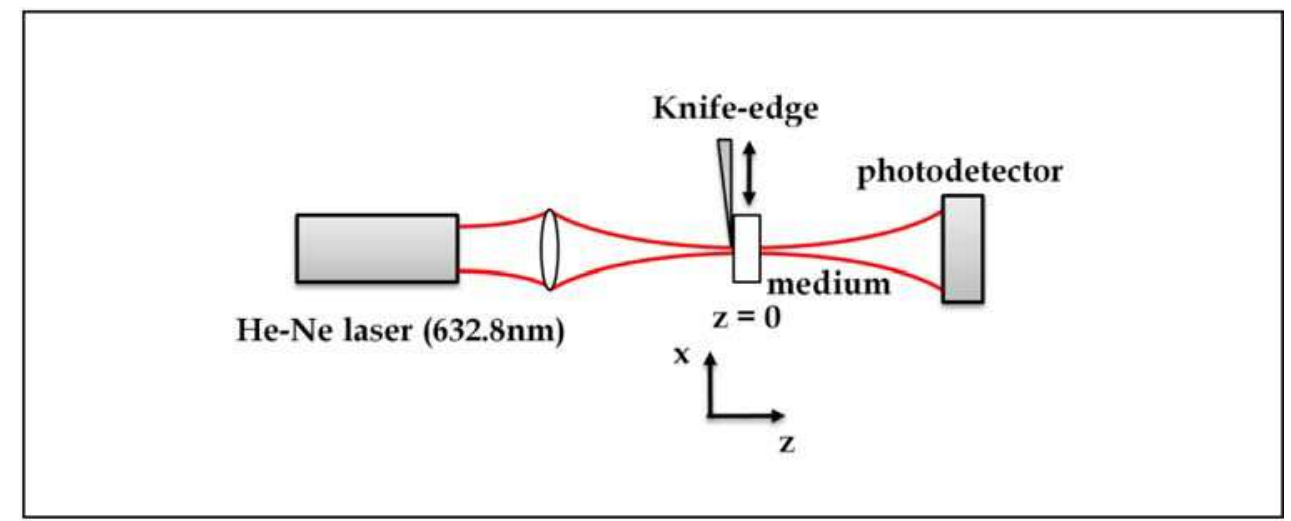

Fig. 1. Experimental setup for knife-edge $\mathrm{X}$-scan technique for measuring nonlinear absorption.

The knife edge is positioned in front of a nonlinear optical medium placed at the focus (i.e., $z=0)$ and is transversely scanned to the beam propagation axis from negative infinity to present knife-edge position. In case of two photon absorption process, the variation of beam power for a fundamental Gaussian laser beam passing through the medium can be written as

$$
P\left(x^{\prime}, z\right)=\int_{-\infty}^{\infty} \int_{-\infty}^{x^{\prime}} I_{e}(x, y, z) d x d y
$$

where $I_{e}(x, y, z)$ is the output beam intensity at the exit surface of the sample and is given by Eq.(2a). For a small nonlinear absorbance (i.e., $q(x, y, z)<1$ ), which is valid for our moderate experimental conditions, substituting Eq. (2a) into Eq.(7) with binomial expansion for $q(x, y, z)$ and integrating yields the transmitted power for knife-edge X-scan as 


$$
P\left(x^{\prime}, z\right)=P_{o} \sum_{m=0}^{\infty} \frac{\left(-q_{o}\right)^{m}}{2(m+1)}\left[\operatorname{erf}\left(\sqrt{2(m+1)} x^{\prime} / w(z)\right)+1\right]
$$

where $q_{o}=q(r=0, z)=q_{o o} /\left(1+\tilde{z}^{2}\right), q_{o}=\beta I_{0} L_{c f f}, P_{o}=\pi w_{o}^{2} I_{o} / 2$ is the on-axis power at focus, and $\operatorname{erf}(\bullet)$ is the error function. As is evident from Eq.(8), the first term (i.e., $m=0$ ) is exactly equivalent to the formula for conventional knife-edge scanning without nonlinear sample. The derivative of the transmitted power with respect to $x^{\prime}$ corresponds to a variation of incident Gaussian beam power (i.e., nonlinear Gaussian beam profile) caused by nonlinear absorption and is given by

$$
\frac{d P\left(x^{\prime}, z\right)}{d x^{\prime}}=\frac{P_{o}}{w(z)} \sqrt{\frac{2}{\pi}} \sum_{m=0}^{\infty} \frac{\left(-q_{o}\right)^{m}}{\sqrt{m+1}} \exp \left[-2(m+1) \frac{x^{\prime 2}}{w^{2}(z)}\right] .
$$

Figure 2 represents theoretical curves for normalized transmitted power and its derivative relative to knife-edge position $x^{\prime}$ for various nonlinear absorbance $q_{0}=-0.5$ and +0.5 . Note that the first term (i.e., $m=0$ ) in Eq.(9) reveals one dimensional Gaussian beam power without nonlinear material (i.e., $q_{0}=0$ ) for knife-edge $X$-scan. For negative nonlinear absorption (i.e., $q_{0}<0$ or amplification), the beam radius or full width at half maximum (FWHM) decreases when compared with $q_{0}=0$, while for positive nonlinear absorption (i.e., $q_{0}>0$ or real absorption), the beam radius is much broaden than that of $q_{0}=0$.

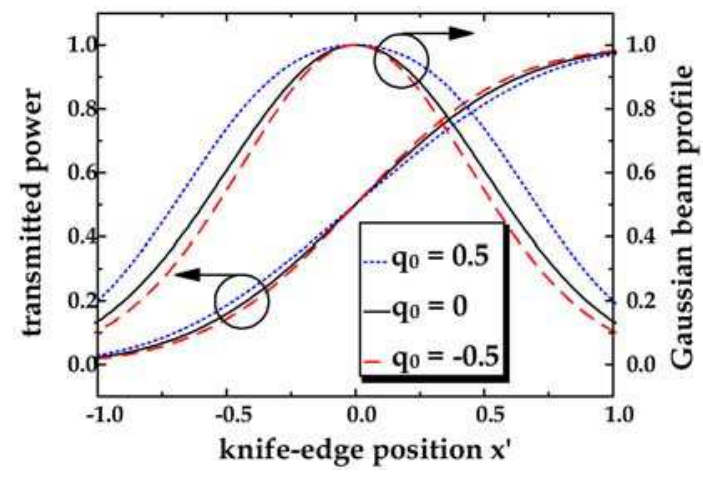

Fig. 2. Theoretical curves for normalized transmitted power and its derivative relative to knife-edge position $x^{\prime}$ (nonlinear Gaussian beam profile) for various nonlinear absorbance.

\subsection{Orientational nonlinear refraction kinetics in nematic liquid crystals for rotational viscosity: Modified closed-aperture Z-scan}

In this section, we will derive the kinetics of orientational refractive index change via director axis torque of nematic liquid crystals (NLCs), which is caused by a Gaussian optical field with/without an applied electric field. We also present a simple and accurate method to measure the rotational viscosity, the response time and the orientational nonlinear refraction in NLCs by modifying the closed Z-scan. Figure 3 shows the experimental setup. The optical method proposed in this work has basically the same experimental geometry used in closed aperture Z-scan. The sole distinction is that the NLC sample is placed at focus 
(i.e., $z=0)$ of an external lens and is fixed at that place during the experiments, unlike Z-scan technique.

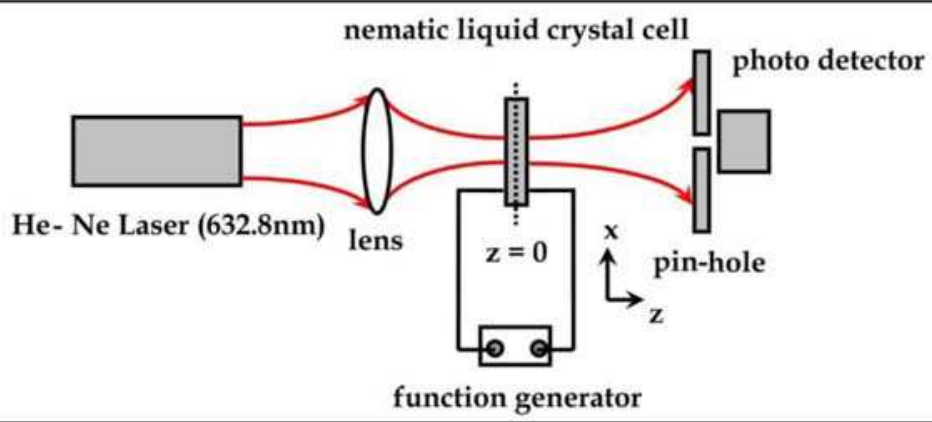

Fig. 3. Schematic diagram of the experimental setup for measuring rotational viscosity of nematic liquid crystal. A rectangular electric field with a pulse duration time $t_{0}$ is applied to the sample.

Before supplying an external electric field by a function generator, a focused optical beam is continuously illuminated to the sample, producing the optical field-induced director axis reorientation (Khoo, 1995), which gives rise to the orientational Kerr effect (OKE) and is given by $\Delta n_{\text {OKE }}(r, z=0)=n_{2, O K E} I_{o} \exp \left[-2 r^{2} / w_{o}^{2}\right]$, where $n_{2, O K E}$ is the nonlinear refractive coefficient for OKE, $I_{0}$ is the on-axis intensity at focus and $w_{0}$ is the beam waist. The on-axis optical intensity of the far-field beam at the aperture plane is measured as a function of time. In this experimental situation, we adopt the closed aperture Z-scan formula, Eq.(5), just by taking $z=0$, which is given by

$$
T_{\text {OKE }}(t)=\frac{1}{1+\frac{4 \eta}{1+q_{\infty}} \Delta \Phi_{o}+\frac{4\left(1+\eta^{2}\right)}{\left(1+q_{\infty}\right)^{2}} \Delta \Phi_{o}^{2}}
$$

Where $\Delta \Phi_{o}=k n_{2, O K E} I_{o} L_{e f f}, q_{\infty o}=\beta I_{o} L_{e f f}$ and $\eta=q_{\infty} / 2 \Delta \Phi_{o}$. When a rectangular electric field with a pulse duration time $t_{0}$ is applied to the sample, the field-induced director axis reorientation will be transient from a non-equilibrium state to an equilibrium state of OKE. In NLCs the field induced reorientation of the director axis is described by a torque balance equation (Khoo, 1995). We define an angle $\theta(r, t)$ as a (small) variation of the director axis orientation angle from stationary director axis angle induced by constant optical field, being spatially and temporally varying. Using the small reorientation angle approximation (i.e., $|\theta|<<1$ ) with the one elastic constant $K$, the torque balance equation is given by (Khoo, 1995; Kim et al., 2004; Kim et al., 2008)

$$
\gamma_{1} \frac{\partial \theta(r, t)}{\partial t}=K\left(\frac{\partial^{2} \theta(r, t)}{\partial x^{2}}+\frac{\partial^{2} \theta(r, t)}{\partial z^{2}}\right)+\left|\vec{\Gamma}_{E}\right|
$$

where $\gamma_{1}$ is the rotational viscosity coefficient, $\left|\vec{\Gamma}_{E}\right|=\Delta \varepsilon \varepsilon_{o}|\hat{n} \cdot \vec{E}(\hat{n} \times \vec{E})| / 4 \pi$ is the magnitude of the director axis torque, which is induced by the applied electric field $\vec{E}_{\text {appl. }}(t)$ and the optical 
electric field $\vec{E}_{\text {opt. }}(r, t)$, and $\hat{n}$ is a unit vector parallel to the reoriented director axis of NLCs, where $\Delta \varepsilon=\varepsilon_{\|}-\varepsilon_{\perp}$ is the dielectric anisotropy. The $\hat{n}$ and the total external electric field $\vec{E}(r, t)=\vec{E}_{\text {appl. }}(r, t)+\vec{E}_{\text {opt. }}(r)$ are expressed as $\hat{n}=(\sin \theta, 0, \cos \theta)$ and $\vec{E}(r, t)=\left(E_{\text {opt. }}, 0, E_{\text {appl. }}\right)$. Then, the director axis torque is approximated as $\left|\vec{\Gamma}_{E}\right|=\Delta \varepsilon \varepsilon_{o}\left(E_{\text {appl. }} E_{\text {opt. }}-\left(E_{\text {appl. }}^{2}-E_{\text {opt. }}^{2}\right) \theta\right) / 4 \pi$ for $|\theta|<<1$. In order to avoid the complexity for solving the equation, we assume that the NLC sample is placed at focus, so the Gaussian optical field can be considered as spatially uniform plane wave. The applied electric field is also spatially uniform and the variation of reorientation angle $\theta(r, t)$ is so small that $\theta(r, t)$ being considered as negligibly small for the secondorder spatial derivatives, but temporally varying. Furthermore, when $E_{\text {appl. }}>E_{\text {opt. }}$, we take the direction of $\theta(t)$ is directed toward the direction of $\vec{E}_{a p p l .}(t)$, whereas for the case of when $E_{\text {appl. }}<E_{\text {opt. }}$ the direction of $\theta(t)$ is directed away from the direction of $\vec{E}_{\text {appl. }}(t)$. With this in mind, Eq.(11) can then be simplified as

$$
\begin{aligned}
& \frac{d \theta(t)}{d t}+\frac{b^{2}-1}{\tau} \theta(t)=\frac{b}{\tau}, \text { for } E_{\text {appl. }}>E_{\text {opt. }} \text { or } b>1 \\
& \frac{d \theta(t)}{d t}+\frac{1-b^{2}}{\tau} \theta(t)=-\frac{b}{\tau}, \text { for } E_{\text {appl. }}<E_{\text {opt. }} \text { or } b<1
\end{aligned}
$$

where $b=E_{\text {appl. }} / E_{\text {opt. }}$ and $\tau=4 \pi \gamma_{1} / \Delta \varepsilon \varepsilon_{0} E_{\text {opt. }}^{2}$ is a response time of NLCs, which is linearly proportional to the rotational viscosity coefficient and is inversely proportional to the optical beam intensity. Consider a rectangular electric pulse with a pulse width $t_{0}$ and an amplitude of $E_{a p p l .}=$ const.is suddenly applied to the sample at time $t=0$ (i.e., $E_{\text {appl. }}(t)=E_{\text {appl. }}=$ const. for $0<t<t_{o}$ otherwise $\left.E_{\text {appl. }}(t)=0\right)$, while an optical field is continuously illuminated the sample from $t=-\infty$ to $t=\infty$. For the case of $b>1$ (i.e., $E_{\text {appl. }}>E_{\text {opt. }}$ ), Eqs.(12) becomes

$$
\begin{gathered}
\frac{d \theta(t)}{d t}+\frac{b^{2}-1}{\tau} \theta(t)=\frac{b}{\tau}, \quad \text { for } 0<t<t_{0} \\
\frac{d \theta(t)}{d t}+\frac{\theta(t)}{\tau}=0, \quad \text { for } t>t_{\circ} .
\end{gathered}
$$

Eqs.(13) can be readily solved by using the boundary conditions of which $\theta(t)$ is continuous at $t=t_{o}$ and $\theta(t=0)=0$, and the solution is given by

$$
\begin{gathered}
\theta\left(t<t_{o}\right)=\frac{b}{b^{2}-1}\left(1-\exp \left[-\frac{b^{2}-1}{\tau} t\right]\right), \text { for } 0<t<t_{o} \\
\theta\left(t \geq t_{o}\right)=\frac{b}{b^{2}-1}\left(1-\exp \left[-\frac{b^{2}-1}{\tau} t_{o}\right]\right) \exp \left[-\frac{t-t_{o}}{\tau}\right] \text { for } t>t_{o} .
\end{gathered}
$$

Similary, for the case of $b<1$ (i.e., $E_{\text {appl. }}<E_{\text {opt. }}$ ), Eqs.(12) becomes 


$$
\begin{gathered}
\frac{d \theta(t)}{d t}+\frac{1-b^{2}}{\tau} \theta(t)=-\frac{b}{\tau}, \text { for } 0<t<t_{o} \\
\frac{d \theta(t)}{d t}+\frac{\theta(t)}{\tau}=0, \text { for } t>t_{0} .
\end{gathered}
$$

The solution to Eqs.(15) is given by

$$
\begin{gathered}
\theta\left(t<t_{o}\right)=-\frac{b}{1-b^{2}}\left(1-\exp \left[-\frac{1-b^{2}}{\tau} t\right]\right), \text { for } 0<t<t_{o} \\
\theta\left(t \geq t_{o}\right)=-\frac{b}{1-b^{2}}\left(1-\exp \left[-\frac{1-b^{2}}{\tau} t_{o}\right]\right) \exp \left[-\frac{t-t_{o}}{\tau}\right] \text { for } t>t_{o} .
\end{gathered}
$$

Since the orientational refractive index is proportional to $\theta(t)$ (Khoo, 1995), defining the transient orientational nonlinear refractive index as $\Delta n_{\text {trans. }}(t) \equiv \delta n_{\text {trans. }} \theta(t)$ where $\delta n_{\text {trans. }}$ is a proportional constant, mainly depending on the dielectric anisotropy, then the total orientational nonlinear refraction consists of the transient contribution, $\Delta n_{\text {trans. }}(t)$ owing to the transient electric field and the stationary contribution, $\Delta n_{O K E}$ due to the constant optical field. Therefore, the total nonlinear phase shift $\Delta \Phi_{o}(t)$, experienced by the optical beam in travelling the NLC sample is given by $\Delta \Phi_{o}(t)=k\left[n_{2, O K E} I_{o}+\delta n_{\text {trans }} \theta(t)\right] L_{c f f}$, where $L_{\text {eff }}=\left(1-\exp \left[-\alpha_{0} L\right]\right) / \alpha_{0}$. Figure 4 represents the theoretical predictions of the transient optical transmittance of Eq.(10) and the reorientation angle of the director axis of Eqs.(14) in NLC sample. In this simulation, we use the following parameters: $\Delta \varepsilon=10, E_{\text {opt }}=0.1 \mathrm{~V} / \mu \mathrm{m}$ $E_{\text {appl }}=0.5 \mathrm{~V} / \mu \mathrm{m}, b=5, q_{\infty o}=0.5, \eta=10^{-2}, \gamma_{1}=10^{-1} \mathrm{~Pa} \cdot \mathrm{s}$ and $t_{o}=100 \mathrm{~ms}$, respectively.

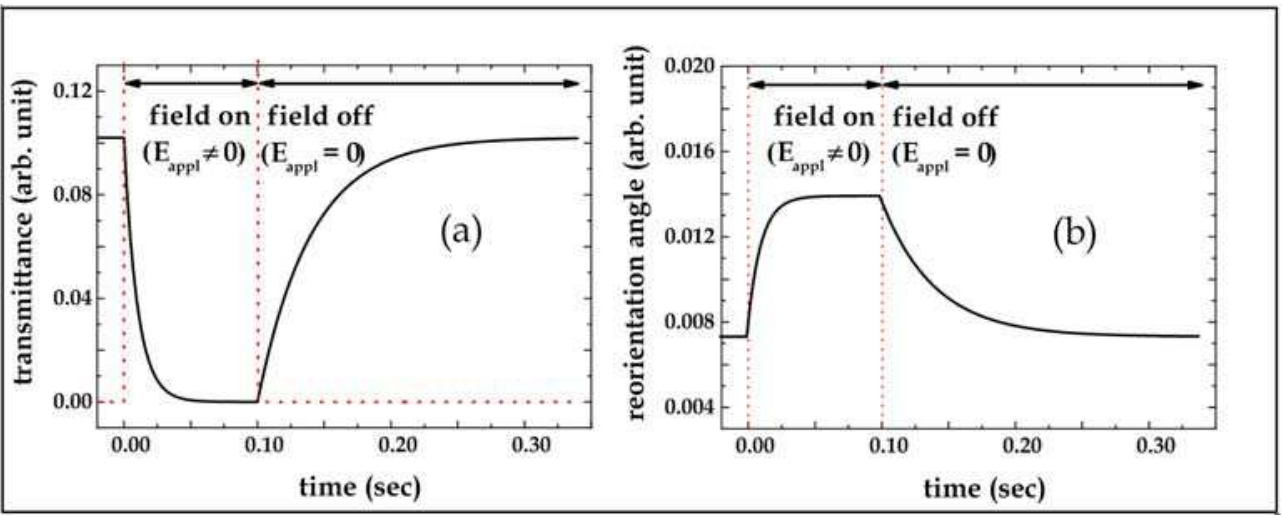

Fig. 4. Theoretical curves of (a) normalized transmittance and (b) reorientation angle as a function of time.

\section{Experiments and discussions}

\subsection{Sample preparation of nematic liquid crystals cell}

We fabricated porphyrin:Zn-doped nematic liquid crystal (NLC) cells filled by capillary phenomenon between two transparent indium-tin-oxide coated glass substrates with $20 \mu \mathrm{m}$ 
thick beads as a spacer. Two glass substrates were assembled by UV bond and then filled inside of cells with porphyrin:Zn-doped nematic liquid crystal for various concentrations of dye $(0,0.006,0.13,0.50 w t \%)$. The liquid crystal used was the eutectic liquid crystal mixture, commercially known as E7 (Merck Ltd.), which has a positive dielectric anisotropy $\Delta \varepsilon=13.8$, the elastic constants $K_{11}=1.11 \times 10^{-11} N$ and $K_{33}=1.71 \times 10^{-11} \mathrm{~N}$ at room temperature and wavelength $\lambda=589 \mathrm{~nm}$. Zn-doped porphyrin dye [5, 10, 15, 20-tetraphenylporphyrinatozinc (ZnTPP)] was supplied by Busan National University. We made no surface treatments to NLC sample, so the director axis orientations are random before they are subject to any optical field or applied electric field.

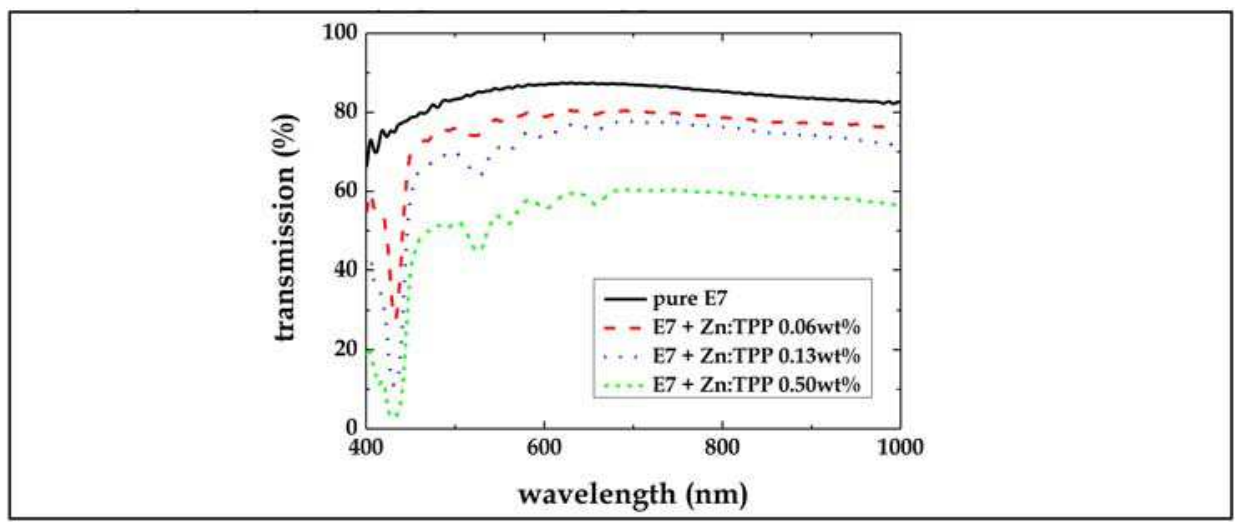

Fig. 5. Transmission spectra for various dye concentrations of porphyrin:Zn in nematic E7 liquid crystal.

The transmission spectrum for pure E7 NLC cell reveals nearly transparent of about $90 \%$ in visible wavelength range, as shown in Fig. 5. As increasing the concentrations of dye the transmisstion spectrum is gradually decreased. It is also shown from Fig. 5 that Zn-doped porphyrin dye is photosensitive to blue-green wavelength region. The linear absorption coefficients for various dye concentrations at wavelength $632.8 \mathrm{~nm}$ were estimated by using the Beer-Lambert law $\left(T=\exp \left[-\alpha_{0} L\right]\right)$, neglecting the Fresnel reflection at surfaces of the sample as follows: $\alpha_{\text {pure }}=0.676 \times 10^{2} \mathrm{~cm}^{-1}, \alpha_{0.06 \text { wt } \%}=1.095 \times 10^{2} \mathrm{~cm}^{-1}, \alpha_{0.13 \mathrm{wt} \%}=1.315 \times 10^{2} \mathrm{~cm}^{-1}$, and $\alpha_{0.50 w t}=2.607 \times 10^{2} \mathrm{~cm}^{-1}$.

\subsection{Determinations of nonlinear absorption coefficient by using knife-edge $X$-scan and open-aperture Z-scan}

In this section, we determine the nonlinear absorption coefficients for various dye concentrations in NLC sample by means of knife-edge X-scan method and open-aperture Zscan method and compare the experimental results quantitatively. Figure 1 represents the schematic diagram for the knife-edge X-scan method. The cw He-Ne laser of wavelength $\lambda=632.8 \mathrm{~nm}$ is used for experiments and the laser beam power is $3 \mathrm{~mW}$. The focal length of biconvex lens is $20 \mathrm{~cm}$. The whole transmitted power is measured by a photo detector during the knife-edge scan. Before conducting the knife-edge X-scan experiment, we have to determine the incident Gaussian laser beam profiles such as beam radius $w(z)$, beam waist $w_{0}$ and radius of curvature of the wave front $R(z)$ at $z$. Figure 6(a) shows the typical experimental results of normalized power for knife-edge scan against scan $x^{\prime}$ distance at 
several, which are well fitted with the theoretic formula as $P\left(x^{\prime}, z\right) / P_{o}=\left[\operatorname{erf}\left(\sqrt{2(m+1)} x^{\prime} / w(z)\right)+1\right] / 2$. Figure $6(\mathrm{~b})$ represents the beam radius extracted from Fig.6(a) with theoretical curve, yielding the beam waist $w_{0}=4.90 \mu \mathrm{m}$, the onaxis intensity at focus $I_{0}=8.0 \mathrm{~kW} / \mathrm{cm}^{2}$ and the optical field $E_{\text {optc. }}=0.22 \mathrm{~V} / \mu \mathrm{m}$.
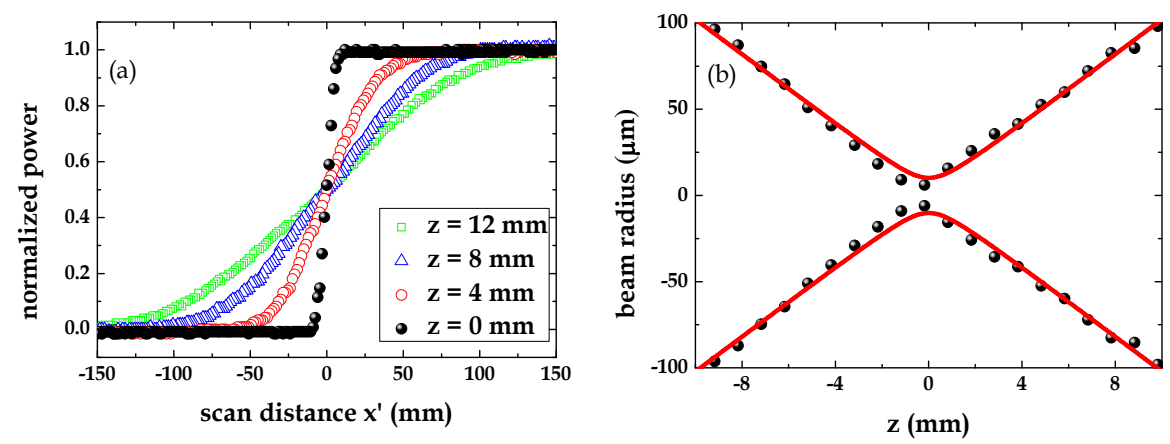

Fig. 6. (a) The measured laser beam power for knife-edge scanning vs. scan distance $x^{\prime}$, at several fixed $z$ positions and (b) the measured Gaussian beam radius $w(z)$ with theoretical curve.

To determine the nonlinear absorption coefficient of the sample we performed two kinds of experiments; one is the knife-edge X-scan in which the sample is placed at rear face of the knife-edge, as shown in Fig. 1, and the other is the conventional open-aperture Z-scan. Since the closed-aperture Z-scan transmittance is entangled with the nonlinear refraction and the nonlinear absorption, as described in Eq.(5), one should determine the nonlinear absorption coefficient before finding the nonlinear refractive coefficient. Once the nonlinear absorption coefficient $\beta$ is extracted from the open aperture Z-scan or the knife-edge X-scan, one can extracts the remaining unknown nonlinear refractive coefficient $n_{2}$ from the closed aperture Z-scan transmittance. Figure 7 represents the typical experimental results of the knife-edge $\mathrm{X}$-scan and the open aperture Z-scan for various dye concentrations with the theoretical predictions.

Table 1 compares the nonlinear absorption coefficient $\beta$ for various dye concentrations, determined by the knife-edge X-scan method with the open aperture Z-scan method. Nonlinear absorption coefficients determined by two methods are in good agreement with each other.

\begin{tabular}{|c|c|c|}
\hline $\begin{array}{c}\text { Dye concentration } \\
{[w t \%]}\end{array}$ & $\begin{array}{c}\text { Knife-edge X-scan } \\
\beta(\mathrm{cm} / \mathrm{kW})\end{array}$ & $\begin{array}{c}\text { Open aperture Z-scan } \\
\beta(\mathrm{cm} / \mathrm{kW})\end{array}$ \\
\hline 0 & 1 & 3 \\
\hline 0.06 & 38 & 33 \\
\hline 0.13 & 72 & 75 \\
\hline 0.50 & 286 & 300 \\
\hline
\end{tabular}

Table 1. Comparison of knife-edge X-scan with open aperture Z-scan for determined nonlinear absorption coefficients for various dye concentrations of nematic liquid crystal. 


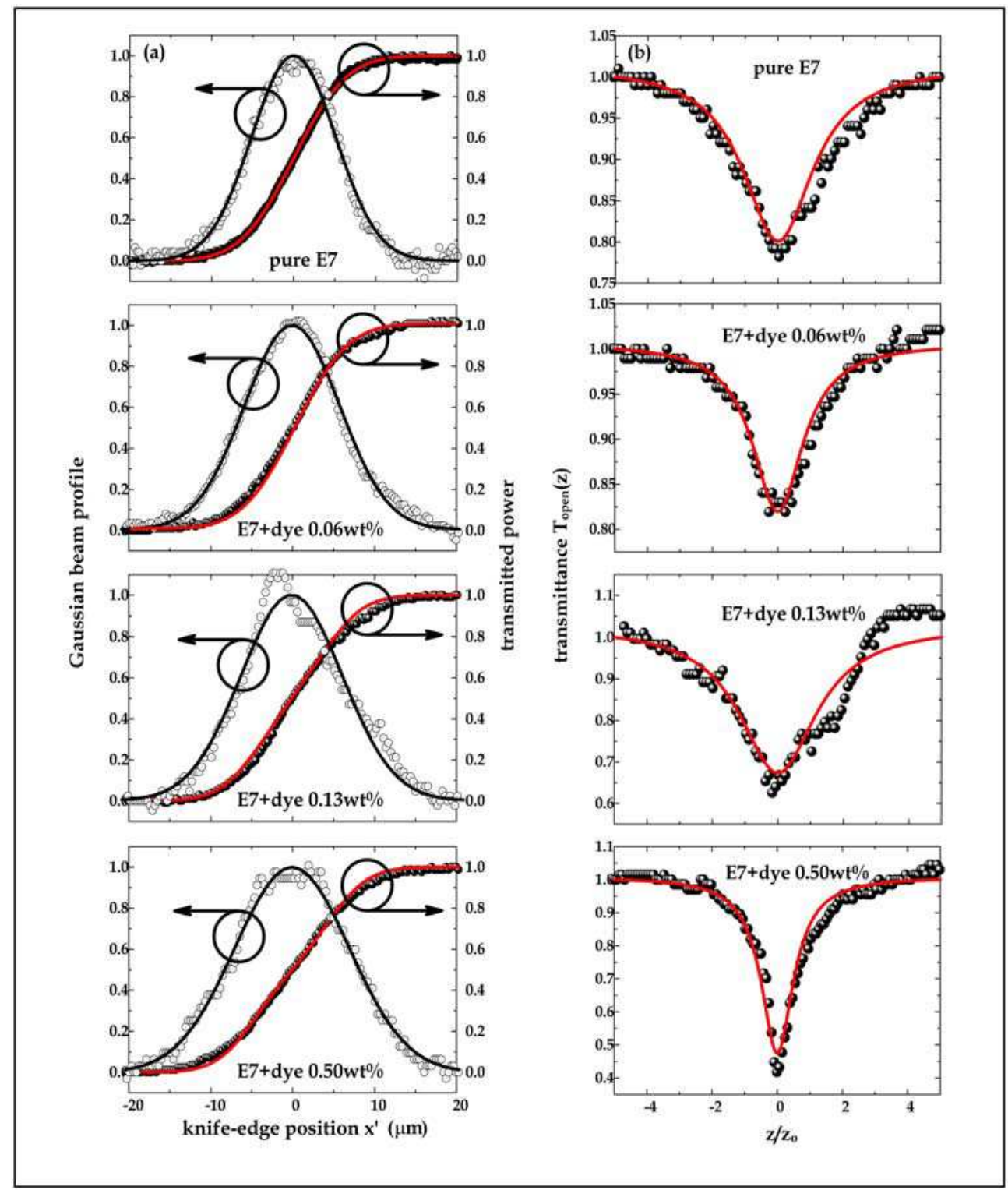

Fig. 7. (a) Knife-edge X-scan data and (b) open aperture Z-scan data for various dye concentrations of nematic liquid crystal with theoretical curves. 


\subsection{Determinations of nonlinear refractive coefficient by using closed aperture Z-scan}

Figure 8 depicts the typical closed aperture Z-scan data, revealing a self-defocusing nature. The nonlinear refractive coefficients are determined from the best curve fitting using Eq.(5) with the known nonlinear absorption coefficients obtained from preceding subsection.

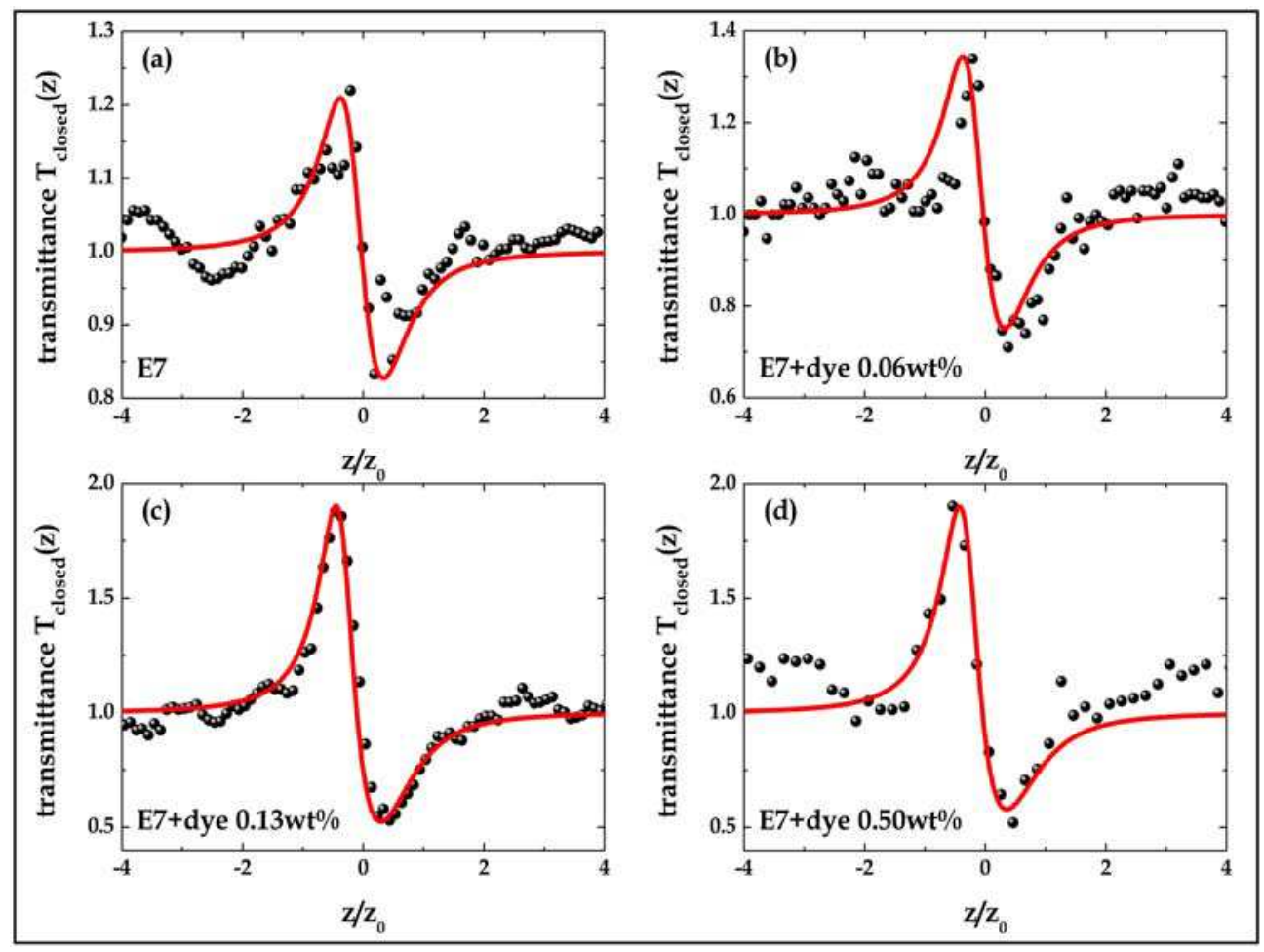

Fig. 8. The closed-aperture Z-scan transmittance data for various dye concentrations of nematic liquid crystal with theoretic curves of Eq.(5).

\subsection{Determinations of rotational viscosity by modified closed-aperture Z-scan}

Following the method described in subsection 2.3, we conducted the transient optical transmittance experiments by applying the rectangular electric field with the pulse duration time of $t_{o}=30 \mathrm{~ms}$ and the amplitude of $E_{\text {appl. }}=0.5 \mathrm{~V} / \mu \mathrm{m}$. The NLC sample is placed at focus (i.e., $z=0$ ) of an external lens and is fixed at that place during the experiments. The optical field is $E_{\text {opt. }}=0.22 \mathrm{~V} / \mu \mathrm{m}$ at focus (i.e., $z=0$ ), so $b=E_{\text {appl. }} / E_{\text {opt. }}=2.3$. Before applying the rectangular pulse field, the focused optical beam which is continuously illuminated produces a bias nonlinear refraction, which is called the optical-field induced orientational 


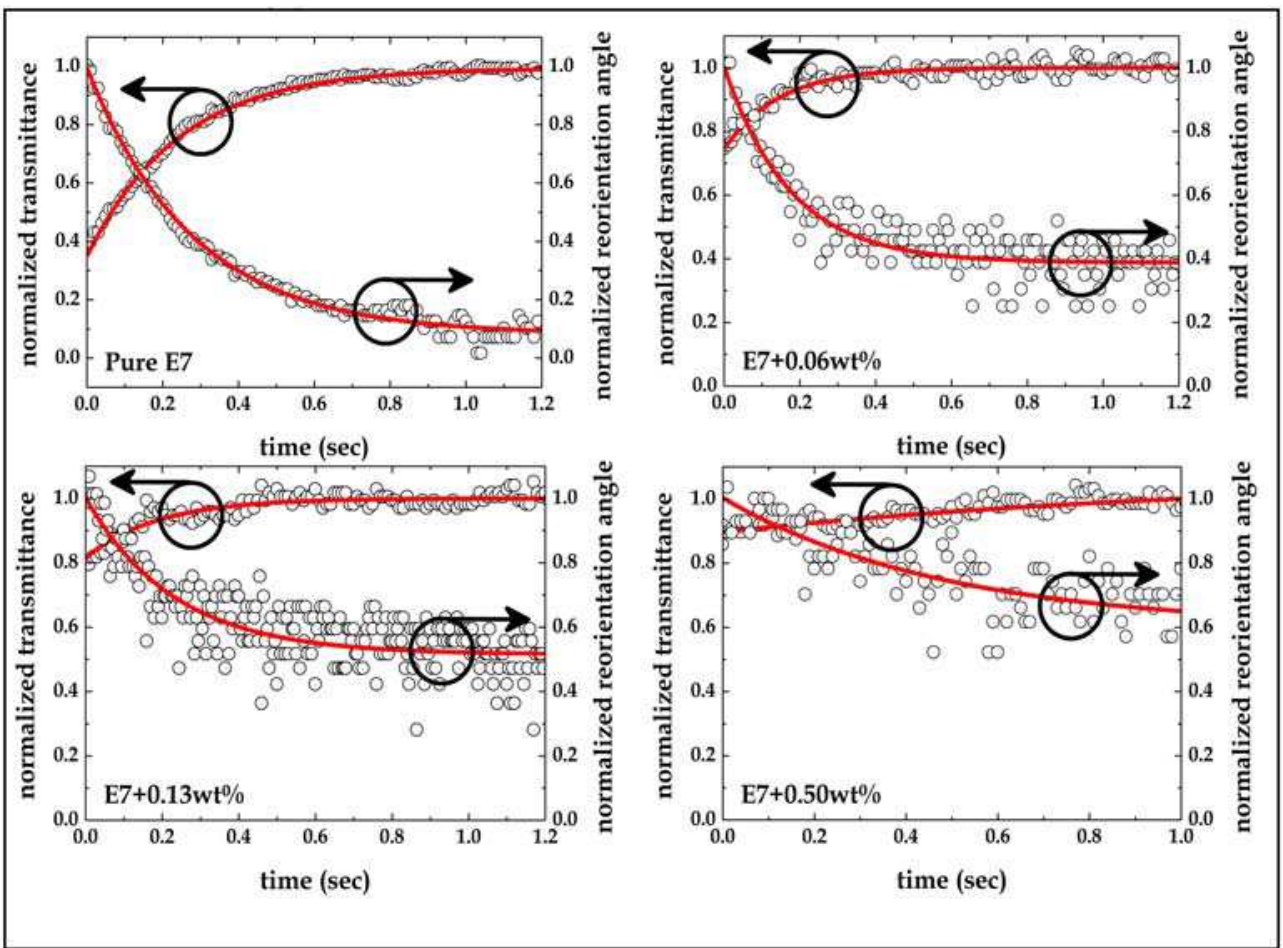

Fig. 9. The experimental data of normalized transient optical transmittance and normalized reorientation angle against time for various dye concentrations with theoretical curves.

Kerr effect (OKE), $\Delta n_{\mathrm{OKE}}=n_{2, \text { OKE }} I_{o}$. Figure 9 represents the transient optical transmittances and the corresponding reorientation angles for various dye concentrations after the electric field is switched off. It should be noted that the steady state value of the reorientation angles do not go to zero, strongly implying the existence of the bias OKE caused by constant optical field, as theoretically predicted.

Table 2 compares the rotational viscosity coefficient and nonlinear refractive index coefficient for various dye concentrations, determined by the transient optical transmittance (or modified Z-scan) method with the closed aperture Z-scan method. Nonlinear refractive index coefficient, which is determined by two methods are in good agreement with each other. It reveals that the physical mechanism of the Kerr effect in NLC is caused by the optical field-induced director axis reorientation. It is also noted that the measured value of the rotational viscosity coefficient of $0.23 \mathrm{~Pa} \cdot \mathrm{s}$ for pure E7 is almost the same value of $0.224 \mathrm{~Pa} \cdot \mathrm{s}$ at $25^{\circ} \mathrm{C}$ by means of transient current method (Chen \& Lee, 2007). 


\begin{tabular}{|c|c|c|c|}
\hline $\begin{array}{c}\text { Dye } \\
\text { concentration } \\
{[w t \%]}\end{array}$ & $\begin{array}{c}\text { Rotational } \\
\text { viscosity } \\
\text { coefficient } \\
\gamma_{1}(\mathrm{~Pa} \cdot \mathrm{s})\end{array}$ & $\begin{array}{c}\text { Modified closed } \\
\text { aperture Z-scan } \\
n_{2, \text { oKE }}\left(\mathrm{cm}^{2} / \mathrm{MW}\right)\end{array}$ & $\begin{array}{c}\text { Closed aperture } \\
\text { Z-scan } \\
n_{2}\left(\mathrm{~cm}^{2} / \mathrm{MW}\right)\end{array}$ \\
\hline 0 & 0.23 & -0.40 & -0.42 \\
\hline 0.06 & 0.16 & -0.85 & -0.94 \\
\hline 0.13 & 0.20 & -1.60 & -1.47 \\
\hline 0.50 & 0.39 & -4.27 & -4.46 \\
\hline
\end{tabular}

Table 2. Rotational viscosity coefficient and nonlinear refractive coefficient for various dye concentrations.

\section{Conclusion}

In this chapter, we propose simple and accurate optical methods to determine the nonlinear refraction, the nonlinear absorption and the rotational viscosity coefficient in dye-doped nematic liquid crystals, and also develop the corresponding theories. The versatile optical methods presented are as follows: (i) closed aperture Z-scan for measuring both the sign and the magnitude of the optical nonlinear refraction, taking into accounting two photon absorption, (ii) open aperture Z-scan and knife-edge X-scan for measuring the nonlinear absorption coefficient unambiguously, and (iii) modified closed aperture Z-scan (or transient optical transmittance) method by applying a rectangular electric field to measure the orientational Kerr effect (OKE) and the rotational viscosity coefficient. The measured values of optical nonlinearities and the rotational viscosity by an optical method are crosschecked by another method, showing excellent agreement with each other.

\section{References}

Chen, H. Y.; Lee, W. \& Clark N. A. (2007). Faster electro-optical response characteristics of a carbon-nanotube-nematic suspension. Appl. Phys. Lett., Vol 90, Issue 3, 033510, ISSN: 0003-6951

deSouza, P. C.; Nader, G.; Catunda, T.; Muramatsu, M. \& Horowicz, R. J. (1999). Application of the Z-scan technique to a saturable photorefractive medium with the overlapped ground and excited state absorption. Opt. Comm., Vol. 177, Issue 1-6, 417-423, ISSN: 0030-4018

Durand, G.; Leger, L.; Rondelez, F. \& Veyssie, M. (Orsay Liquid Crystal Group) (1995). Quasielastic Rayleigh scattering in nematic liquid crystals. Phys. Rev. Lett., Vol. 22, Issue 25, 1361-1363, ISSN: 0031-9007

Gomes, A. S. L.; Demenicis, L.; Petrov, D. V.; de Araujo, C. B.; de Melo, C. P. \& Souto-Maior, R. (1996). Time-resolved picosecond optical nonlinearity and all-optical Kerr gate in poly (3-hexadecylthiophene). Appl. Phys. Lett., Vol. 69, Issue 15, 2166-2168, ISSN : 0003-6951

Imai, M.; Naito, H.; Okuda, M. \& Sugimura, A. (1995). Determination of rotational viscosity and pretilt angle in nematic liquid crystals from transient current: influence of ionic conduction. Mol. Cryst. Liq. Cryst., Vol. 259, 37-46, ISSN: 1542-1406 
Khoo, I. C. (1995). Liquid crystals: Physical properties and nonlinear optical phenomena. 121-150, John Wiley \& Sons, Inc., ISBN: 0-471-30362-3, New York

Kneppe, H.; Schneider, F. \& Sharma, N. K. (1982). Rotational viscosity of nematic liquid crystals. J. Chem. Phys. Vol. 77, Issue 6, 3203-3208, ISSN: 0021-9606 1

Kim, E. J.; Yang, H. R.; Lee, S. J.; Kim, G. Y. \& Kwak, C. H. (2008). Orientational photorefractive holograms in porphyrin:Zn-doped nematic liquid crystals. Opt. Express, Vol. 16, Issue 22, 17329-17341, ISSN: 1094-4087

Kim, K. H.; Kim, E. J.; Lee, S. J.; Lee, J. H.; Kim, J. E. \& Kwak, C. H. (2004). Effects of applied field on orientational photorefraction in porphyrin:Zn-doped nematic liquid crystals. Appl. Phys. Lett., Vol. 85, Issue 3, 366-368, ISSN: 0003-6951

Kwak, C. H.; Lee, Y. L. \& Kim, S. G. (1999). Analysis of asymmetric Z-scan measurement for large optical nonlinearities in an amorphous As2S3 thin film. J. Opt. Soc. Am. B, Vol. 16, Issue 4, 600-604, ISSN: 0740-3224

Leenhouts, F. (1985). Determination of the rotational viscosity from the director pattern relaxation in twisted nematic cells. J. Appl. Phys., Vol. 58, Issue 6, 2180-2183, ISSN: 0021-8979

Lenz, G.; Zimmermann, J.; Katsufuji, T.; Lines, M. E.; Hwang, H. Y.; Spalter, S.; Slusher, R. E.; Cheong, S. W.; Sangher, J. S. \& Aggarwal, I. D. (2000). Large Kerr effect in bulk Se-based chalcogenide glasses. Opt. Lett., Vol. 25, Issue 4, 254-256, ISSN: 01469592

Lefkiry, M.; Phu, X. N. and Rivoire, G. (1998). Existence of a bistable polarization state in a Kerr medium in the presence of two-photon absorption. Quantum Semiclass. Opt., Vol. 10, No. 1, 283-292 ISSN: 1464-4266

Liao, H. B.; Xiao, R. F.; Wang, H.; Wong, K. S. \& Wong, G. K. L. (1998). Large third-order optical nonlinearity in $\mathrm{Au}: \mathrm{TiO}_{2}$ composite films measured on a femtosecond time scale. Appl. Phys. Lett., Vol. 72, Issue 15, 1817-1819, ISSN: 0003-6951

Martinoty, P. \& Candau, S. (1971). Determination of viscosity coefficents of a nematic liqid crystal using a shear waves relectance technique. Mol. Cryst. Liq. Cryst., Vol. 14 243271, ISSN: 1542-1406

Martins, A. F.; Esnault, P. \& Volino, F. (1986). Measurement of the viscoelastic coefficients of main-chain nematic polymers by an NMR technique. Phys. Rev. Lett., Vol. 57, Issue 14, 1745-1748, ISSN: 0031-9007

Prost, J. \& Gasparoux, H. (1971). Determination of twist viscosity coefficient in the nematic mesophases Phys. Lett. A, Vol. 36, Issue 3, 245-256, ISSN: 0375-9601

Sheik-Bahae, M.; Said, A. A.; Wei, T. H.; Hagan, D. J. \& Stryland, E. V. (1990). Sensitive measurement of optical nonlinearities using a single beam. IEEE J. Quantum Electron., Vol. 26, Issue 4, 760-769, ISSN: 0018-9197

Suzaki, Y. \& Tachibana, A. (1975). Measurement of the $\mu \mathrm{m}$ sized radius of Gaussian laser beam using the scanning knife-edge. Appl. Opt., Vol. 14, Issue 12, 2809-2810, ISSN: 0003-6935

Watanabe, Y.; Ohnishi, M. \& Tsuchiya, T. (1995). Measurement of nonlinear absorption and refraction in titanium dioxide single crystal by using a phase distortion method. Appl. Phys. Lett., Vol 66, Issue 25, 3431-3432, ISSN: 0003-6951 
Yavrian, A.; Galstyan, T. V. \& Pich'e, M. (1999). Single-mirror interferometer for nonlinear optical characterization. IEEE J. Quantum Electron., Vol. 35, 1430-1433, ISSN: 00189197 


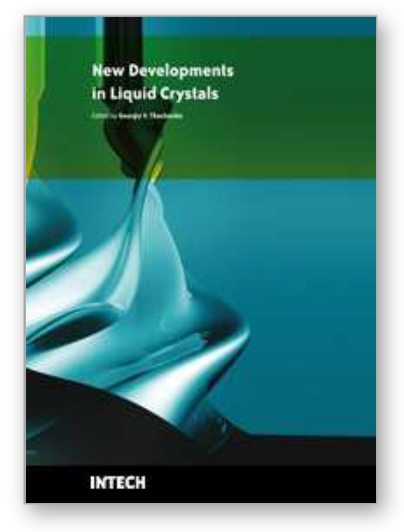

\author{
New Developments in Liquid Crystals \\ Edited by Georgiy V Tkachenko
}

ISBN 978-953-307-015-5

Hard cover, 234 pages

Publisher InTech

Published online 01, November, 2009

Published in print edition November, 2009

Liquid crystal technology is a subject of many advanced areas of science and engineering. It is commonly associated with liquid crystal displays applied in calculators, watches, mobile phones, digital cameras, monitors etc. But nowadays liquid crystals find more and more use in photonics, telecommunications, medicine and other fields. The goal of this book is to show the increasing importance of liquid crystals in industrial and scientific applications and inspire future research and engineering ideas in students, young researchers and practitioners.

\title{
How to reference
}

In order to correctly reference this scholarly work, feel free to copy and paste the following:

Gun Yeup Kim and Chong Hoon Kwak (2009). Simple Optical Methods for Measuring Optical Nonlinearities and Rotational Viscosity in Nematic Liquid Crystals, New Developments in Liquid Crystals, Georgiy V Tkachenko (Ed.), ISBN: 978-953-307-015-5, InTech, Available from: http://www.intechopen.com/books/newdevelopments-in-liquid-crystals/simple-optical-methods-for-measuring-optical-nonlinearities-and-rotationalviscosity-in-nematic-liqu

\section{INTECH}

open science | open minds

\section{InTech Europe}

University Campus STeP Ri

Slavka Krautzeka 83/A

51000 Rijeka, Croatia

Phone: +385 (51) 770447

Fax: +385 (51) 686166

www.intechopen.com

\section{InTech China}

Unit 405, Office Block, Hotel Equatorial Shanghai

No.65, Yan An Road (West), Shanghai, 200040, China

中国上海市延安西路65号上海国际贵都大饭店办公楼405单元

Phone: +86-21-62489820

Fax: +86-21-62489821 
(C) 2009 The Author(s). Licensee IntechOpen. This chapter is distributed under the terms of the Creative Commons Attribution-NonCommercialShareAlike-3.0 License, which permits use, distribution and reproduction for non-commercial purposes, provided the original is properly cited and derivative works building on this content are distributed under the same license. 